\title{
CLINICAL FEATURES AND RISK FACTORS OF AUTOIMMUNE LIVER INVOLVEMENT IN PEDIATRIC INFLAMMATORY BOWEL DISEASE
}

Matteo Bramuzzo MD, ${ }^{1}$ Stefano Martelossi MD, ${ }^{1}$ Giuliano Torre MD,${ }^{2}$ Sabrina Cardile MD, ${ }^{2}$ Serena Arrigo MD, ${ }^{3}$ Silvia Vignola MD ${ }^{3}$ Federica Ferrari MD, ${ }^{4}$ Giovanna Zuin MD, Maria Teresa Illiceto MD, ${ }^{6}$ Marco Gasparetto MD, ${ }^{7}$ Salvatore Pellegrino MD, PhD, ${ }^{8}$ Claudio Romano MD, ${ }^{9}$ Giuseppe Maggiore MD, ${ }^{10}$ Marcella Montico MSc, ${ }^{11}$ Marina Aloi MD, $\mathrm{PhD}^{4}$ On behalf of the SIGENP IBD Group

\section{Affiliations}

1 Paediatric Department, Gastroenterology and Nutrition Unit, Institute for Maternal and Child Health, IRCCS “Burlo Garofolo”, Trieste, Italy.

2 Hepatology, Gastroenterology and Nutrition Unit, Bambino Gesù Children's Hospital, Rome, Italy.

3 Paediatric Gastroenterology Unit, Institute “Giannina Gaslini”, Genoa, Italy.

4 Paediatric Gastroenterology and Liver Unit, Sapienza University of Rome, Rome, Italy.

5 Paediatric Department, Children's Hospital "V. Buzzi," Milan, Italy.

6 Unit of Paediatric Gastroenterology and Digestive Endoscopy, Department of Paediatrics Hospital "S.Spirito", Pescara, Italy.

7 Paediatric Gastroenterology Unit, Department of Women and Children's Health, Padua University Hospital, Padua, Italy.

8 Paediatric Gastroenterology and Cystic Fibrosis Unit, University of Messina, Messina, Italy.

9 Paediatric Gastroenterology and Endoscopy, Regional Center for IBD, Paediatric Department, University of Messina, Messina, Italy.

10 Paediatric Gastroenterology and Hepatology, University of Pisa, Pisa, Italy.

11 Clinical Epidemiology and Public Health Research Unit, Institute for Maternal and Child Health, IRCCS “Burlo Garofolo”, Trieste, Italy. 


\section{Corresponding author}

Marina Aloi, MD, PhD

Paediatric Gastroenterology and Liver Unit

Department of Paediatrics

Viale Regina Elena 324

00161 Rome (Italy)

Tel. +390649979387

Fax +390649979387

e-mail:marina.aloi@uniroma1.it 


\section{Conflicts of interest and source of funding}

The Authors declare no conflict of interest and no funding for their work.

Word count: 3088; number of figures: 1; number of tables: 3 .

Matteo Bramuzzo: contributed to design the work, to acquire, to analyse and to interpret the data; drafted the manuscript and approved the final version of the manuscript.

Stefano Martelossi: contributed to design the work, to acquire and to interpret the data; revised the work for important intellectual content and approved the final version of the manuscript.

Giuliano Torre: contributed to acquire the data; revised the work for important intellectual content and approved the final version of the manuscript.

Sabrina Cardile: contributed to acquire the data; revised the work for important intellectual content and approved the final version of the manuscript.

Serena Arrigo: contributed to acquire the data; revised the work for important intellectual content and approved the final version of the manuscript.

Silvia Vignola: contributed to acquire the data; revised the work for important intellectual content and approved the final version of the manuscript.

Federica Ferrari: contributed to acquire the data; revised the work for important intellectual content and approved the final version of the manuscript.

Giovanna Zuin: contributed to acquire the data; revised the work for important intellectual content and approved the final version of the manuscript.

Maria Teresa Illiceto: contributed to acquire the data; revised the work for important intellectual content and approved the final version of the manuscript.

Marco Gasparetto: contributed to acquire the data; revised the work for important intellectual content and approved the final version of the manuscript.

Salvatore Pellegrino: contributed to acquire the data; revised the work for important intellectual content and approved the final version of the manuscript. 
Claudio Romano: contributed to acquire the data; revised the work for important intellectual content and approved the final version of the manuscript.

Giuseppe Maggiore: contributed to design the work and to acquire the data; revised the work for important intellectual content and approved the final version of the manuscript.

Marcella Montico: contributed to analyse and to interpret the data; revised the work for important intellectual content and approved the final version of the manuscript.

Marina Aloi: contributed to design the work and to acquire the data; revised the work for important intellectual content and approved the final version of the manuscript.

All authors ensure the accuracy and the integrity of the data.

\section{Abstract}

Background. Autoimmune liver disease is reported in up to $7.8 \%$ of children with inflammatory bowel disease. A distinct inflammatory bowel disease phenotype has been suggested in adults and in small pediatric cohorts.

Aims. To evaluate the features of inflammatory bowel disease associated with autoimmune liver diseases and to analyze the characteristics of the liver disease.

Methods. Information on patients was obtained from the Italian Pediatric Inflammatory Bowel Disease Registry. Data of patients with and without autoimmune liver disease were compared. Results. Autoimmune liver disease was detected in $6.8 \%$ of the 677 patients enrolled and was significantly associated with the diagnosis of ulcerative colitis (83\%), with pancolonic involvement $(84 \%)$, and with perinuclear anti-neutrophil cytoplasmic antibody positivity $(41 \%)\left(\right.$ all $\left.\mathrm{p}_{\mathrm{s}}<0.05\right)$. Autoimmune liver disease was defined as sclerosing cholangitis in $61 \%$ of the patients and as an overlap syndrome in $33 \%$. Concomitant intra and extrahepatic biliary involvement was reported in 
$61 \%$ of cases, while exclusive extrahepatic lesions were reported in $21 \%$. Hepatobiliary complications were observed in $9 \%$ of the patients during follow-up.

Conclusions. Autoimmune liver disease, especially sclerosing cholangitis, was significantly more common in patients with extensive ulcerative colitis. Although complications are relatively rare in the pediatric age, monitoring is recommended.

Key words: children; inflammatory bowel disease; sclerosing cholangitis; autoimmune hepatitis; overlap syndrome 


\section{What is known}

- Up to $8 \%$ of children with inflammatory bowel disease (IBD) have autoimmune liver disease (AILD).

- Adult patients with AILD typically present with an ulcerative pancolitis, rectal sparing and backwash ileitis.

\section{What is new}

- Children with IBD and AILD seem to have a milder disease course less often needing a surgical management than those with isolated IBD.

- A relatively higher number of children present exclusively with extrahepatic involvement.

\section{List of abbreviations}

AIH: autoimmune hepatitis; AILD: autoimmune liver disease; ALT: alanine aminotransferase; ANA: anti-nuclear antibody; ANCA: anti-neutrophil citoplasmic antibody; ASCA: antiSaccaromyces cerevisiae antibody; CD: Crohn's disease; GGT: gamma-glutamyl transferase; IBD: inflammatory bowel disease; IBDU: inflammatory bowel disease unclassified; LKM1: antiliver/kidney microsomal type1 antibody; SC: sclerosing cholangitis; SMA: smooth muscle antibody; UC: ulcerative colitis; UDCA: ursodexossicolic acid. 


\section{INTRODUCTION}

Approximately $20 \%$ of children with inflammatory bowel disease (IBD) present with extraintestinal manifestations, ${ }^{1}$ likely sharing an immune-dysregulatory origin with the intestinal disease. ${ }^{1}$

Autoimmune liver disease (AILD) has been reported in up to $7.8 \%$ of young patients with IBD ${ }^{2,3}$ and may present with sclerosing cholangitis (SC), autoimmune hepatitis (AIH) or, when features of both SC and AIH exist, as "overlap syndrome". SC is a progressive inflammatory fibro-obliterative disorder that may affect intra or extrahepatic bile ducts while AIH is a chronic progressive inflammatory process of the liver parenchyma, characterized by interface hepatitis, specific autoantibody positivity and increased immunoglobulin G levels. Both SC and AIH associated with IBD are supposed to be autoimmune disorders, triggered, in genetically susceptible patients, by environmental factors such as pathogens or toxin translocation from the gut to the portal system, migration of intestinal activated leukocytes and secondary alterations to bile composition. ${ }^{4,5}$ Since the features of both biliary tree sclerosis and interface hepatitis may coexist or develop from each other, it has also been postulated that SC and AIH may be two manifestations of the same pathogenic process. ${ }^{4}$ In adult populations, IBD with associated AILD seems to be distinguished by a particular phenotype. First, ulcerative colitis (UC) is the most frequent type of IBD diagnosed and pancolitis with rectal sparing and backwash ileitis is the most commonly described phenotype. ${ }^{6,7}$ Moreover, although diffuse, the intestinal inflammation in AILD-associated IBD is generally mild, with longer remission periods and fewer relapses than in isolated IBD. ${ }^{8,9}$ However, there is a reported higher risk of pouchitis after colectomy and ileoanal anastomosis ${ }^{10}$ and an increased risk not only of cholangiocarcinoma $^{5,11}$ but also of colorectal cancer. ${ }^{5,9}$

Little data exists for children and whether pediatric IBD with AILD have distinguishing features is still debated. ${ }^{12}$

In order to assess the distinguishing features and the risk factors for the association between IBD and AILD in children, we performed a cohort study and compared data of patients with an AILD- 
associated IBD and IBD patients without AILD. Secondly, to evaluate the characteristics of the AILD-associated with IBD, we analyzed its clinical and radiological features.

\section{METHODS}

Patients were identified from the Italian Pediatric IBD Registry, established by the Pediatric Gastroenterology, Hepatology and Nutrition Italian Society (SIGENP), which prospectively collects demographic and clinical data of patients less than 18 years old with a new diagnosis of IBD from the whole national territory. Children are enrolled in the Registry at diagnosis, after parental consent; medical information from electronic or paper charts is entered into the registry by trained investigators.

Data of children enrolled and stored in the registry from January $1^{\text {st }}, 2009$ to December $31^{\text {st }}, 2014$ (the data retrieval date) were used for this study. Eligible subjects were patients with any form of IBD (UC, Crohn's disease - CD, and inflammatory bowel disease unclassified - IBDU). Diagnosis of IBD and its classification were based on clinical history, physical examination, endoscopic appearance, histologic findings, and radiologic studies, according to the Porto criteria. ${ }^{13}$ The most recent visit before the retrieval date was considered as the last follow-up. Only patients with a length of follow-up for the IBD more than 6 months were included for the analysis. The information retrieved for the purpose of this study included: i) demographic features: sex, age at diagnosis of IBD, duration of IBD symptoms before diagnosis, family history for IBD, extraintestinal manifestations; ii) IBD phenotype characteristics: type of IBD, disease distribution and behaviour, auto-antibodies pattern; iii) need for third line medical therapies and surgery. A positive family history for IBD was defined by the presence of IBD in a first-degree relative. Extraintestinal manifestations included hepatobiliary, skin, joint and ocular manifestations and other autoimmune diseases, if reported. 
Disease distribution and behaviour were collected at the time of diagnosis and during follow-up according to the Paris Classification. ${ }^{14}$ The most extensive and complicated disease phenotype was used for comparison. Phenotypic changes were also collected and analyzed.

IBD-specific autoantibodies included: Anti-Neutrophil Cytoplasmic antibodies (ANCA) and AntiSaccharomyces Cerevisiae antibodies (ASCA).

Need for third line therapies, including infliximab, adalimumab, cyclosporine and thalidomide, or surgery were evaluated in order to assess disease severity at follow-up. Information regarding AILD characteristics was obtained from specific questionnaires requested from the participating centres. These included: age at AILD diagnosis, presence of hepatobiliary symptoms (itching, asthenia, anorexia, epigastric pain) at AILD diagnosis, elevation of serum alanine aminotransferase (ALT) and gamma-glutamyl transpeptidase (GGT) greater than 45 UI/L, type of AILD, autoantibodies associated to liver or biliary disease (anti-nuclear - ANA, anti-smooth muscle - SMA, antiliver/kidney microsomal type 1 - LKM1), location of the biliary disease based on radiographic imaging and histological findings from liver biopsies, AILD treatment, evolution and complications (acute cholangitis, portal hypertension, hepatic failure, cholangiocarcinoma, death).

Laboratory and imaging exams were performed at each centre and were not centralized.

Data on other autoantibodies such as anti-liver cytosol type 1 and anti-soluble liver antigen/liver pancreas were not collected because they are not considered indispensable for the diagnosis and the classification of the AILD ${ }^{15}$ and they are not routinely tested in the pediatric centres.

AILD was classified as: SC, AIH or overlap syndrome. The diagnosis of SC was based on biochemical cholestatic liver profiles, presence of strictures and dilatations of bile ducts and on the histological findings of inflammatory fibro-obliterative damage to the biliary ducts. ${ }^{4},{ }^{16}$ AIH was defined by the presence of elevated serum transaminase and gamma globulin levels, ANA, SMA or LKM1 positivity and by the histological finding of "interface hepatitis" or "bridging collapse" in absence of other cause of hepatitis, according to the criteria defined by the International AIH Group ${ }^{17}$ and adapted for children by Mieli-Vergani et al. ${ }^{16}$ No scoring system was used 
because of the lack of pediatric standards. An overlap syndrome was defined as an "autoimmune sclerosing cholangitis", and the diagnosis was made when characteristics of both SC and AIH were present $^{4,16}$

\section{Statistical Methods}

Categorical variables are presented as absolute numbers, percentages, and odds ratios (ORs) with 95\% confidence intervals (CIs). Continuous variables are reported as median and ranges.

Comparisons between IBD patients with AILD and patients without AILD were performed using the t-Student test for continuous variables and the Fisher exact test for categorical variables.

Logistic regression analysis was performed to assess the relationship between patient characteristics and the presence of AILD, adjusting for type of IBD. Multivariate logistic regression analysis was used to access variables independently associated with AILD. Variables associated with a p-value of less than 0.2 were considered for inclusion in the model. A backward stepwise approach was used to identify variables independently associated with AILD with a p-value of less than 0.05. Two models were built, one for IBD and one for UC. All statistical tests were 2-sided. A p value of 0.05 or less was considered significant. The analysis was carried out using Stata 11.2.

\section{RESULTS}

Nine hundred and twenty patients were identified in the registry at the retrieval date. Six hundred and seventy-seven patients met the criteria for inclusion: 46 (6.8\%) presented with AILD and were considered as the study population. The remaining $631(93.2 \%)$ were used as controls.

The median follow-up time was similar between patients with AILD [2.8 (0.5-5.7) years] and IBD controls [2.7 (0.5 -5.9) years].

There were no significant differences in sex, age at the time of IBD diagnosis, duration of intestinal symptoms before the diagnosis and family history, between patients with AILD and controls. Also, no differences were detected when adjusting the analysis for the IBD type (table 1). 
IBD type distribution differed significantly between patients with AILD and IBD controls (figure 1). UC was the most significant feature associated with AILD [38/46 (83\%) versus 322/631 (51\%), adjusted OR 6.8, 95\% CI 2.6-17.4]. A detailed comparison of patients with AILD-UC and UC controls is reported in table 2.

A diagnosis of IBDU was reported in $3(6 \%)$ patients with AILD and in $22(3 \%)$ without (adjusted OR $7.8,95 \%$ CI $1.7-34.9)$.

Five patients with CD and AILD presented with no significant differences in disease location and behaviour compared to $287 \mathrm{CD}$ controls. All patients in the AILD group had ileal involvement [2 were classified as L1 (distal ileum with or without limited cecal localization), 3 as L3 (ileocolonic)] and presented with an inflammatory behaviour at IBD onset; one evolved into a stenosingpenetrating phenotype during the follow-up; none had perianal disease.

No significant differences were reported for the IBD type during the course of the disease [4/46 (9\%) AILD and 32/631 (5\%) controls]. When evaluating all IBD patients, a significant association was found between ANCA positivity and IBD with AILD [17/42 (40\%) versus 38/231 (16\%) IBD controls, OR 3.4, 95\% CI 1.7 - 7.0] and between ASCA positivity and IBD without AILD [2/40 (10\%) versus $72 / 244(30 \%)$, OR $0.1,95 \%$ CI $0.0-0.5]$. ANCA positivity in patients with UC is reported in table 2; ASCA was positive in 1/5 (20\%) patients with CD and AILD and in 27/109 $(25 \%)$ patients tested $(\mathrm{p}=1.0)$

No significant difference was found for third-line therapy use [13/46 (28\%) AILD and 200/631 (32\%) controls]. The overall need for surgery was lower in AILD patients than in IBD controls [0/46 (0\%) AILD and 75/631 (12\%) controls, $\mathrm{p}<0.05)$.

In the multivariate logistic regression analysis, the diagnosis of UC and the ANCA positivity were independent predictors of the presence of AILD (OR 4.9, 95\% CI 1.8-13.3 and OR 2.6, 95\% CI 1.25.6, respectively). In UC, ANCA positivity and pancolitis were the only factors identified (OR 2.5, 95\% CI 1.1-5.8 and OR 3.2, 95\% CI 1.1-9.2, respectively). 


\section{Analysis of the AILD characteristics}

Of 46 patients with AILD, $28(61 \%)$ were diagnosed as SC, $3(6 \%)$ as AIH and $15(33 \%)$ as overlap syndrome. Diagnosis was based on magnetic resonance cholangiography and bioptic findings in 33 (72\%), ultrasound and bioptic findings in $6(13 \%)$, magnetic resonance cholangiography alone in 6 (13\%), and endoscopic retrograde cholangiopancreatography and bioptic findings in $1(2 \%)$. AILD was diagnosed at the same time as IBD in $25(54 \%)$ patients, before in $10(22 \%)$, with a median time interval of 6.5 months (range 1-84), and after in $11(24 \%)$, with a median delay of 6 months (range 1-25 months). Clinical symptoms attributable to the hepatobiliary disease (asthenia, anorexia, epigastric pain) were found in $12(26 \%)$ patients; only $1(2 \%)$ patient reported itching; at diagnosis, liver enzymes were elevated in $41(89 \%)$ patients, normal in $2(4 \%)$ and not specified in $3(7 \%)$

Once patients with AIH were excluded, in the remaining 43 patients with biliary alterations, both intra and extrahepatic branches were involved in 31 (72\%) while exclusive extrahepatic involvement was reported in $9(21 \%)$ and exclusive intrahepatic in $3(7 \%)$.

ANA was positive in 27/41 (64\%) patients, SMA in 20/37 (54\%), and LKM1 in 1/29 (3\%).

All patients were treated with ursodeoxicholic acid and 39 (85\%) with azathioprine; $8(17 \%)$

received second line or experimental therapies for the AILD: 3 (7\%) cyclosporine, 3 (7\%) mofetil mycophenolate and $2(4 \%)$ oral vancomycin.

The median follow up time for AILD was 2.8 years (range 0.5-11.5). During this period 1 patient developed acute cholangitis, portal hypertension and hepatic failure and required liver transplantation, 2 had episodes of bacterial cholangitis, and 1 developed signs of portal hypertension (table 3). 


\section{DISCUSSION}

Our study focused on patients with AILD identified in a population of children with IBD and evaluated the risk factors associated to the hepatobiliary disease through the comparison with an IBD control group.

We found that AILD occurred in $6.8 \%$ of children with IBD, consistent with a prevalence of 6.4 to $7.8 \%$ reported in the literature from the United States, ${ }^{1,3,18}$ and of $6.5 \%$ reported in a Finnish cohort. $^{19}$

In our cohort we did not find any demographic characteristics to be related with AILD.

Although previous studies reported AILD-UC and AILD-CD patients to be more commonly male and female, respectively, ${ }^{20,21}$ we did not find any significant association with gender. No correlation between AILD and age at the diagnosis of IBD and duration of symptoms emerged from our analysis. This result is in keeping with previous data in adults. ${ }^{22}$

Our study confirms a significant association between AILD and UC $(\mathrm{p}<0.05)$, with nearly two thirds of patients with a diagnosis of AILD receiving a diagnosis of UC. Consistent with previous data, which report AILD in $12 \%$ of patients with an underlying diagnosis of UC and $1.8 \%$ with CD patients, ${ }^{3}$ we found AILD in $11 \%$ and $2 \%$ of UC and CD patients, respectively. Surprisingly, although not statistically significant and based on small numbers, a high proportion of patients with AILD received a diagnosis of IBDU.

In UC, an extensive colonic disease was significantly more common in patients with combined AILD which is similar to data already reported in adults ${ }^{7}$ and in a small pediatric series. ${ }^{12,23}$ It was not possible to collect and analyze the pattern of segmental colonic inflammation. Thus no conclusion can be drawn on the more severe inflammatory involvement of the right colon as reported in patients with AILD. ${ }^{5}$

As far as CD is concerned, previous studies suggest a predominance of the colonic involvement ${ }^{23,24}$ and a lower prevalence of strictures and perianal disease in adults. ${ }^{25}$ However our small sample of patients with CD and AILD did not allow us to make any comparisons with the current literature. 
Our study found ANCA positivity to be a significant risk factor for the association between IBD and AILD.

A high prevalence of ANCA in patients with AILD regardless of the presence of IBD has been previously reported, ${ }^{26}$ and it may be speculated that IBD and AILD share common pathogenetic pathways that determine the development of ANCA. However our results should be interpreted with caution due to the lower prevalence of ANCA positivity than expected in patients with IBD. ${ }^{27}$ IBD with AILD has been reported to have a milder course with less clinical relapses and complications than isolated IBD, but it is still not clear if AILD affects the overall risk of surgery for intestinal disease..$^{22,28}$

In our cohorts, while no difference was found in the use of third line treatments, a surgical intervention was significantly more frequent in isolated IBD, than in IBD with AILD. This result may suggest that, at least in children, AILD-associated intestinal disease may have both a less complicated course and also a reduced risk for intestinal surgery.

Our study also evaluated the prevalence of different types of AILD. Exclusive biliary damage was found in $60.9 \%$ of cases while exclusive parenchymal injury of the liver was detected only in $6.5 \%$ of patients. Overlap syndrome was detected in one third of the cases reinforcing the hypothesis of a strict relation between hepatic and biliary lesions. Unfortunately, our study was not designed to evaluate changes in AILD abnormalities and the risk factors that predispose to the spreading of the damages from one structure to another.

While in the adult population more than $60 \%$ of cases of SC are diagnosed at least one year after the diagnosis of IBD, ${ }^{7}$ in our study more than half the patients were diagnosed with IBD and AILD simultaneously, similar to what is reported in another pediatric study. ${ }^{29}$ Less than one third of the patients complained of symptoms attributable to the hepatobiliary disease. However, these symptoms are nonspecific and can be caused also by the intestinal disease. Only one patient complained of itching, which is a more specific symptom of cholestasis. On the contrary most patients had an elevated ALT or GGT confirming that the measurement of the liver enzymes is the 
most helpful diagnostic tool in the identification of IBD-associated AILD. ${ }^{30}$ In $4 \%$ of cases liver enzymes were normal at the time of AILD diagnosis but hepatobiliary abnormalities were found at imaging.

Our study confirmed that the involvement of both intra and extrahepatic ducts is the most common form of biliary localization in children. ${ }^{29,31}$ However, contrary to what has been reported by other authors, we found a higher prevalence of exclusive extrahepatic biliary lesions than isolated intrahepatic ones; this figure contrasts with the hypothesis that SC would start as an intrahepatic disease and would subsequently spread to the extrahepatic ducts. ${ }^{32}$

Finally, we observed a low rate of AILD-related complications. Nevertheless, serious events with potentially life-threatening evolution may occur not only in adults, but also in children, within a few years from disease onset. In the series by Feldstein et al, during a mean follow-up of 6.6 years, $6 \%$ of patients had episodes of bacterial cholangitis and 13\% manifested with bleeding oesophageal varices in half of the cases. ${ }^{29}$ Deneau et al reported complications in $18-42 \%$ of patients depending on the AILD type, including two cases of cholangiocarcinoma diagnosed 4 and 6 years after the diagnosis of SC, respectively. ${ }^{3}$

The main strength of our study is to be based on a prospective national registry, which allowed us to enrol a large pediatric cohort of patient with IBD and AILD and a large number of controls. Moreover, the structured collection of data regarding the features of the AILD permitted their detailed description. For these reasons it is reasonable to consider our results as generalizable to other pediatric cohorts with IBD.

However our study may have some limitations. First, the classification of the AILD types was not based on a predefined protocol and it may be biased by an inhomogeneous radiological and histological definition. For this reason the number of overlap syndromes, whose diagnosis requires the specific evaluation of the coexistence of biliary damage and of interface hepatitis, may be underestimated. We tried to avoid this bias by checking the agreement between the AILD type reported by the centres and the radiological and histological findings of each single patient. 
Furthermore, because of its design, our study could not directly evaluate the correlation between the natural history of IBD and that of AILD. We used the changes in the IBD phenotype and the therapies administered as surrogates of severity, but we were not able to analyze how they impacted the course of AILD.

In conclusion, our study, which was based on a large cohort of children with IBD, confirms that the diagnosis of UC, especially with pancolonic involvement and ANCA positivity, is the main risk factor for AILD, which in most cases involves damages of the intra and extrahepatic bile ducts with or without a concomitant hepatitis.

AILD-associated IBD has a mild course with a small rate of bowel resection surgery; on the other hand, AILD may develop potential severe hepatobiliary complication already in the paediatric age. As they are often asymptomatic, patients with IBD should always be screened for AILD and followup.

Future studies on the causes and the mechanisms underlying IBD-related AILD would help to plan preventive strategies and therapeutic interventions to monitor the progression of hepatobiliary disease. 


\section{REFERENCES}

1. Jose FA, Garnett EA, Vittinghoff E, et al. Development of extraintestinal manifestations in paediatric patients with inflammatory bowel disease. Inflamm Bowel Dis 2009;15:63-8.

2. Dotson JL, Hyams JS, Markowitz J, et al. Extraintestinal manifestations of paediatric inflammatory bowel disease and their relation to disease type and severity. J Pediatr Gastroenterol Nutr 2010;51:140-5.

3. Deneau M, Jensen MK, Holmen J, et al. Primary sclerosing cholangitis, autoimmune hepatitis, and overlap in Utah children: epidemiology and natural history. Hepatology 2013;58:1392-400.

4. Maggiore G, Riva S, Sciveres M. Autoimmune diseases of the liver and biliary tract and overlap syndromes in childhood. Minerva Gastroenterol Dietol 2009;55:53-70.

5. Hirschfield GM, Karlsen TH, Lindor KD, et al. Primary sclerosing cholangitis. Lancet 2013;382:1587-99.

6. Loftus E V, Harewood GC, Loftus CG, et al. PSC-IBD: a unique form of inflammatory bowel disease associated with primary sclerosing cholangitis. Gut 2005;54:91-6.

7. Boonstra K, Erpecum KJ van, Nieuwkerk KMJ van, et al. Primary sclerosing cholangitis is associated with a distinct phenotype of inflammatory bowel disease. Inflamm Bowel Dis 2012;18:2270-6.

8. Lundqvist K, Broomé U. Differences in colonic disease activity in patients with ulcerative colitis with and without primary sclerosing cholangitis: a case control study. Dis Colon Rectum 1997;40:451-6.

9. Sokol H. Disease activity and cancer risk in inflammatory bowel disease associated with primary sclerosing cholangitis. World J Gastroenterol 2008;14:3497.

10. Penna C, Dozois R, Tremaine W, et al. Pouchitis after ileal pouch-anal anastomosis for ulcerative colitis occurs with increased frequency in patients with associated primary sclerosing cholangitis. Gut 1996;38:234-9. 
11. Loftus E, Sandborn W. Interactions between chronic liver disease and inflammatory bowel disease. Inflamm Bowel Dis 1997;3:288-302.

12. Ordonez F, Lacaille F, Canioni D, et al. Paediatric ulcerative colitis associated with autoimmune diseases: a distinct form of inflammatory bowel disease? Inflamm Bowel Dis 2012;18:1809-17.

13. Levine A, Koletzko S, Turner D, et al. ESPGHAN revised Porto criteria for the diagnosis of inflammatory bowel disease in children and adolescents. J Pediatr Gastroenterol Nutr 2014;58:795-806.

14. Levine A, Griffiths A, Markowitz J, et al. Paediatric modification of the Montreal classification for inflammatory bowel disease: the Paris classification. Inflamm Bowel Dis 2011;17:1314-21.

15. Liberal R, Mieli-Vergani G, Vergani D. Clinical significance of autoantibodies in autoimmune hepatitis. J Autoimmun 2013;46:17-24.

16. Mieli-Vergani G, Vergani D. Paediatric autoimmune liver disease. Arch Dis Child 2013;98:1012-7.

17. Alvarez F, Berg PA, Bianchi FB, et al. International Autoimmune Hepatitis Group Report: review of criteria for diagnosis of autoimmune hepatitis. J Hepatol 1999;31:929-38.

18. Malaty HM, Abraham BP, Mehta S, et al. The natural history of ulcerative colitis in a paediatric population: a follow-up population-based cohort study. Clin Exp Gastroenterol $2013 ; 6: 77-83$.

19. Rönnblom A, Holmström T, Tanghöj H et al. Appearance of hepatobiliary diseases in a population-based cohort with inflammatory bowel diseases (Inflammatory Bowel Disease Cohort of the Uppsala Region). J Gastroenterol Hepatol 2015;30:1288-92.

20. Jose FA, Heyman MB. Extraintestinal manifestations of inflammatory bowel disease. J Pediatr Gastroenterol Nutr 2008;46:124-33. 
21. Halliday JS, Djordjevic J, Lust M, et al. A unique clinical phenotype of primary sclerosing cholangitis associated with Crohn's disease. J Crohns Colitis 2012;6:174-81.

22. Olsson R, Danielsson A, Järnerot G, et al. Prevalence of primary sclerosing cholangitis in patients with ulcerative colitis. Gastroenterology1991;100:1319-23.

23. Faubion WA, Loftus E V, Sandborn WJ, et al. Paediatric "PSC-IBD": a descriptive report of associated inflammatory bowel disease among paediatric patients with psc. J Pediatr Gastroenterol Nutr 2001;33:296-300.

24. Noble-Jamieson G, Heuschkel RB, Torrente F, et al. Colitis-associated sclerosing cholangitis in children: a single centre experience. J Crohns Colitis 2013;7:e414-8.

25. Navaneethan U, G K Venkatesh P, Lashner B a, et al. Severity of primary sclerosing cholangitis and its impact on the clinical outcome of Crohn's disease. J Crohns Colitis 2012;6:674-80.

26. Hov J-R. Autoantibodies in primary sclerosing cholangitis. World J Gastroenterol $2008 ; 14: 3781$.

27. Zholudev A, Zurakowski D, Young W, et al. Serologic testing with ANCA, ASCA, and antiOmpC in children and young adults with Crohn's disease and ulcerative colitis: diagnostic value and correlation with disease phenotype. Am J Gastroenterol 2004;99:2235-41

28. Block M, Jørgensen KK, Oresland T, et al. Colectomy for patients with ulcerative colitis and primary sclerosing cholangitis - what next? J Crohns Colitis 2014;8:421-30.

29. Feldstein AE, Perrault J, El-Youssif M, et al. Primary sclerosing cholangitis in children: a long-term follow-up study. Hepatology 2003;38:210-7.

30. Goyal A, Hyams JS, Lerer T, et al. Liver enzyme elevations within 3 months of diagnosis of inflammatory bowel disease and likelihood of liver disease. J Pediatr Gastroenterol Nutr 2014;59:321-3. 
31. Alexopoulou E, Xenophontos PE, Economopoulos N, et al. Investigative MRI cholangiopancreatography for primary sclerosing cholangitis-type lesions in children with IBD. J Pediatr Gastroenterol Nutr 2012;55:308-13.

32. Gregorio GV, Portmann B, Karani J, et al. Autoimmune hepatitis/sclerosing cholangitis overlap syndrome in childhood: a 16-year prospective study. Hepatology 2001;33:544-53. 


\section{FIGURE LEGENDS}

Figure 1. Inflammatory bowel disease type distribution among patients with and without autoimmune liver disease.

AILD: autoimmune liver disease; IBD: inflammatory bowel disease; IBDU: inflammatory bowel disease unclassified; CD: Crohn's disease; UC: ulcerative colitis.

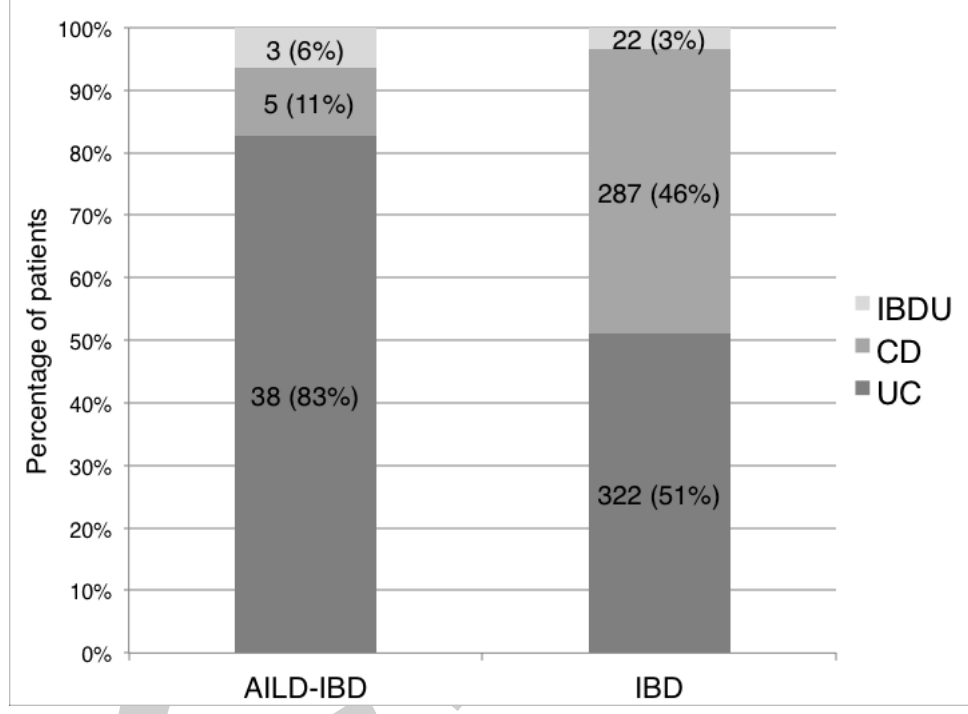


Table 1 . Clinical characteristics of patients with and without autoimmune liver disease.

AILD: autoimmune liver disease; IBD: inflammatory bowel disease.

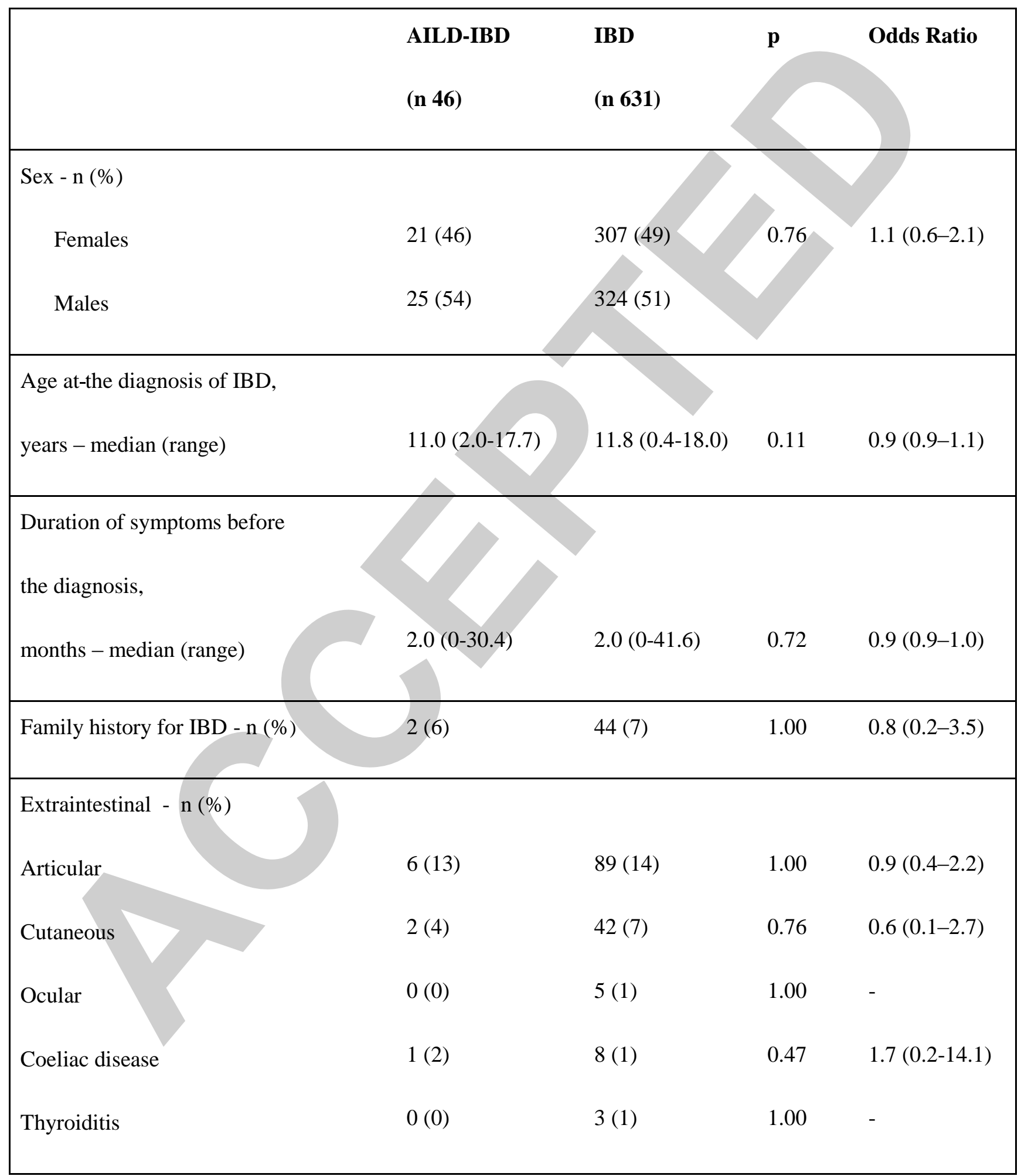


Table 2. Comparison between ulcerative colitis patients with and without autoimmune liver disease.

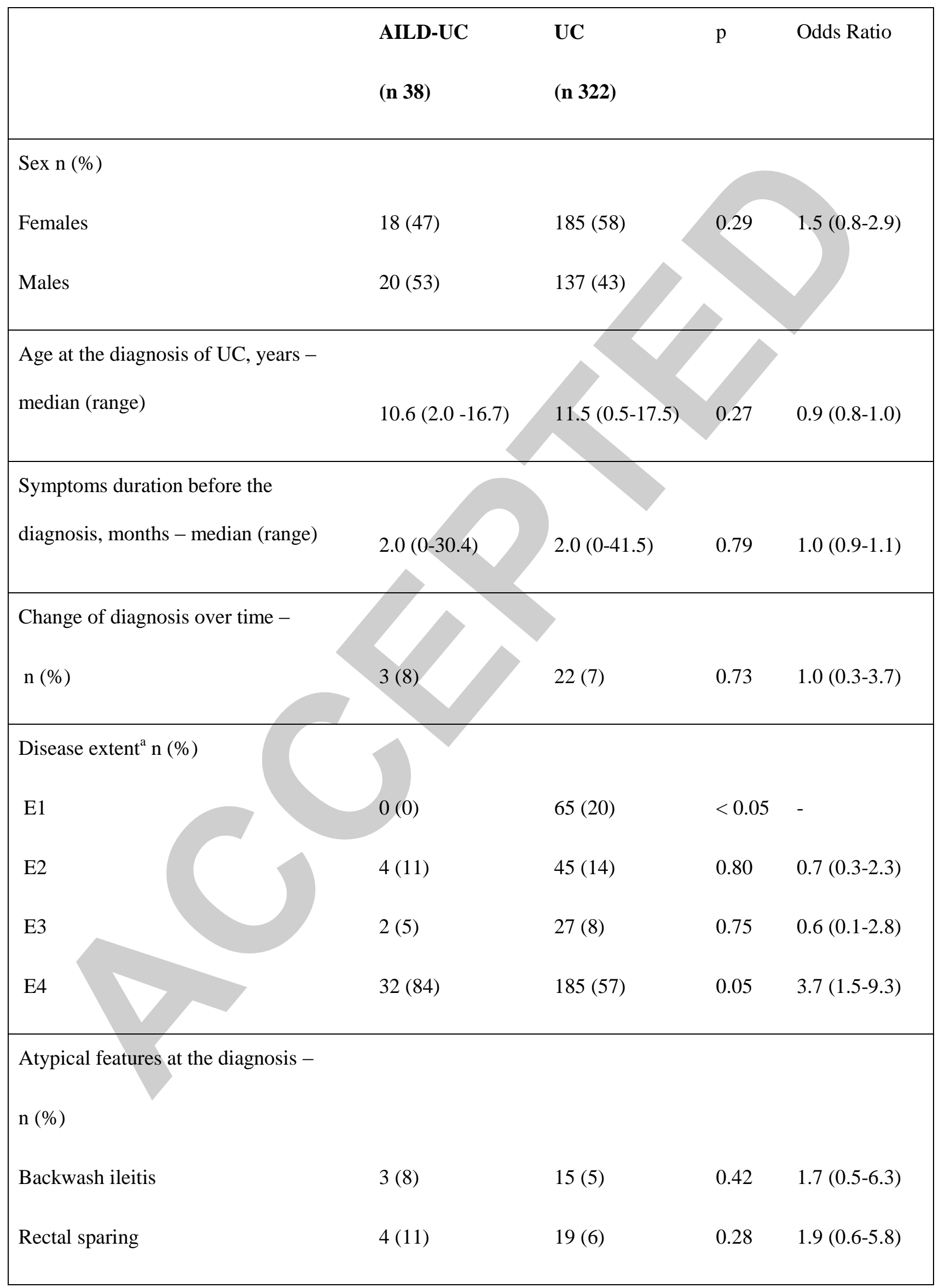




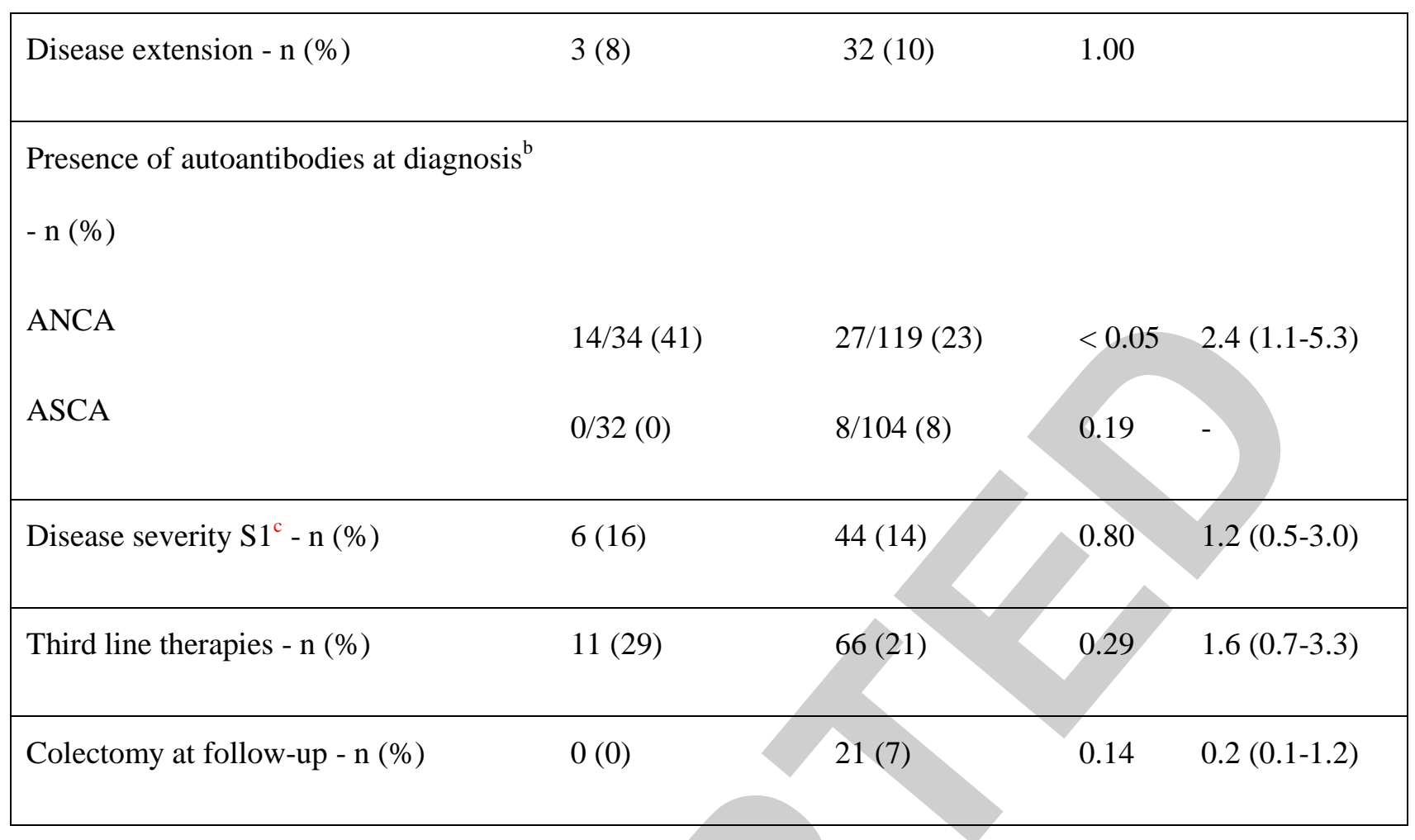

AILD: autoimmune liver disease; UC: ulcerative colitis; ANCA: Anti-Neutrophil Cytoplasmic antibodies; ASCA: AntiSaccharomyces Cerevisiae antibodies

${ }^{\text {a }}$ According to Paris Classification: E1, proctitis; E2 left sided (distal to splenic flexure); E3, extensive (distal to hepatic flexure); E4, pancolitis.

${ }^{\mathrm{b}}$ Autoantibodies were not evaluated in all patients.

${ }^{\mathrm{c}}$ According to Paris Classification: S1 ever severe disease defined as a Pediatric Ulcerative Colitis Activity Index > 65 . 
Table 3. Description of autoimmune liver disease characteristics

\begin{tabular}{|c|c|c|c|}
\hline & $\begin{array}{l}\text { SC } \\
\text { (n 28) }\end{array}$ & $\begin{array}{l}\text { AIH } \\
\text { (n 3) }\end{array}$ & $\begin{array}{l}\text { Overlap syndrome } \\
\text { (n 15) }\end{array}$ \\
\hline \multicolumn{4}{|l|}{ IBD type - n (\%) } \\
\hline $\mathrm{UC}$ & $23(82)$ & $2(67)$ & 3( \\
\hline $\mathrm{CD}$ & $4(14)$ & 0 & $1(7)$ \\
\hline IBDU & $1(4)$ & & $1(7)$ \\
\hline \multicolumn{4}{|l|}{$\operatorname{Sex}-\mathbf{n}(\%)$} \\
\hline Females & $13(46)$ & $2(67)$ & $6(40)$ \\
\hline Males & $15(54)$ & $1(33)$ & $9(60)$ \\
\hline \multicolumn{4}{|l|}{ Diagnosis AILD-IBD - n (\%) } \\
\hline Previous & $8(29)$ & $0(0)$ & $1(7)$ \\
\hline Contemporary & $11(39)$ & $2(67)$ & $12(80)$ \\
\hline Subsequent & $9(32)$ & $1(33)$ & $2(13)$ \\
\hline Hepatobiliary symptoms - n (\%) & $10(36)$ & $0(0)$ & $2(13)$ \\
\hline \multicolumn{4}{|l|}{ Localization biliary tract $-\mathrm{n}(\%)$} \\
\hline Both intra/extra-hepatic & $16(57)$ & - & $15(100)$ \\
\hline Only intrahepatic & $3(11)$ & - & $0(0)$ \\
\hline Only extrahepatic & $9(32)$ & - & $0(0)$ \\
\hline \multicolumn{4}{|l|}{ Autoantibodies $^{\mathrm{a}}$ - n (\%) } \\
\hline ANA & $13 / 24(54)$ & $3 / 3(100)$ & $11 / 15(73)$ \\
\hline
\end{tabular}




\begin{tabular}{|lccc|}
\hline SMA & $10 / 22(45)$ & $1 / 3(33)$ & $9 / 12(75)$ \\
LKM1 & $1 / 18(6)$ & $1 / 3(33)$ & $0 / 8(0)$ \\
\hline Therapies - n (\%) & $28(100)$ & $3(100)$ & $15(100)$ \\
Azathioprine & $23(82)$ & $2(66)$ & $14(93)$ \\
Cyclosporine & $3(11)$ & $0(0)$ & $0(0)$ \\
Mofetil mycofenolate & $3(11)$ & $0(0)$ & $0(0)$ \\
Vancomicine & $2(7)$ & $0(0)$ & $1(7)$ \\
\hline Complications & $1(4)$ & $0(0)$ & $0(0)$ \\
Patients & $1(4)$ & $0(0)$ & \\
Type & $2(7)$ & $0(0)$ & \\
Acute cholangitis & & & \\
\hline
\end{tabular}

${ }^{a}$ Autoantibodies were not evaluated in all cases: the number of patients for whom the data were available is reported for each group.

SC: sclerosing cholangitis; AIH: autoimmune hepatitis; IBD: inflammatory bowel disease; UC: ulcerative colitis; CD: Crohn's disease; IBDU: inflammatory bowel disease unclassified; ANA: anti-nuclear antibodies; SMA anti-smooth muscle antibodies; LKM1: anti-liver/kidney microsomal type 1; UDCA: ursodexossicolic acid. 